\title{
Neck Pain Rehabilitation
}

\author{
Castelli Daniele, Dalila Scaturro, \\ Antonio Sanfilippo, and Giulia Letizia Mauro
}

\section{Definition}

Neck pain is par excellence one of the most common disorders of the musculoskeletal system, second only to low back pain. It constitutes $40 \%$ of all backache. The International Association for the Study of Pain (IASP) defines pain of cervical origin coming from an area between the nuchal line and another imagi-nary line that passes through the lower end of the spinous process of the first thoracic vertebra and the sagittal plans tangent to the side edges of the neck. This definition considers therefore posterior pain which in turn can be divided into high pain, up to $\mathrm{C} 3$, and lower pain, down from C4. Also, as all diseases, it can be divided into acute and chronic neck pain, merely according to the time of onset: it lasts more than 3 months in the first case and for longer in the second case. The painful perception depends on the nociceptive elicitation of the major structures innervated in the neck region, such as cervical muscles, ligaments, facet joints and nerve roots.

The pain may radiate to the upper limbs if there is a nerve root compression, with an annual incidence of 83 per 100,000 individuals, aged between 13 and 91 years, in the US [1]. Clearly the distribution of pain depends on the affected nerve root.

C. Daniele • D. Scaturro • A. Sanfilippo • G.L. Mauro $(\varangle)$ Dipartimento delle discipline chirurgiche,

Oncologiche e stomatologiche,

University of Palermo, Palermo, Italy

e-mail: giulia.letiziamauro@unipa.it

\section{Epidemiology}

Despite being a predominantly benign pathology, it has a significant effect in terms of economic and social costs, due to the significant number of days of absence from work. However in $10 \%$ of cases it tends to become chronic. It affects $30-50 \%$ of the general population every year. It reaches its peak in middle age and women are the most affected [1].

It is estimated that its prevalence is of $12.1-71.5 \%$ in the general population and $27.1-48.8 \%$ in workers, while the annual prevalence of disability is of $1.7-11.5 \%$ [2].

\section{Functional Anatomy}

To understand the importance of this pathology it is necessary to know in detail the cervical spine from the viewpoint of functional anatomy. Cervical spine performs three important functions: it acts as a support for the head, it enables the movements of the head and provides protection to the spinal cord and the vertebral arteries flowing inside. It consists of two anatomically and functionally distinct parts:

- The upper cervical spine, formed by the first two vertebrae (atlas and axis) that are articulated through a three-axis hinge that allows three degrees of freedom; it coordinates in 